\title{
Period Doubling Cascades of Attractors: A Prerequisite for Horseshoes
}

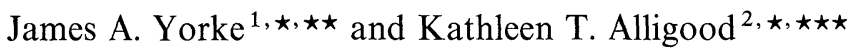 \\ 1 Institute for Physical Science and Technology, and Department of Mathematics, \\ University of Maryland, College Park, MD 20742, USA \\ 2 Department of Mathematics, College of Charleston, Charleston, SC 29424, USA
}

\section{In memory of Charles Conley}

\begin{abstract}
This paper shows that if a horseshoe is created in a natural manner as a parameter is varied, then the process of creation involves the appearance of attracting periodic orbits of all periods. Furthermore, each of these orbits will period double repeatedly, with those periods going to infinity.
\end{abstract}

\section{Introduction}

A two-dimensional horseshoe map is defined on a neighborhood of a rectangle and maps it into a horseshoe shape that lies across the original rectangle, as in Fig. 1c. An analogous map can be defined in higher dimensions. Figure $1 \mathrm{f}$ shows the three dimensional case. Since Smale described horseshoes [S], many dynamical systems have been shown to have them. In the fully developed horseshoe, as described by Smale, there are necessarily immense numbers of periodic orbits: the $k^{\text {th }}$ iterate of the map has $2^{k}$ fixed points. The dynamical behavior in the rectangle is indeed complicated; however, all the periodic orbits there are unstable, and almost any initial point chosen in the rectangle has a trajectory that eventually leaves it.

This paper investigates the dynamical behavior as the horseshoe is being formed in the typical way shown in Fig. 1 . At the initial parameter value $(\lambda=0)$, the rectangle and its image are disjoint; at $\lambda=1$, there is a horseshoe map, with infinitely many unstable periodic points. We assume that for each parameter value,

\footnotetext{
* Research partially supported by the National Science Foundation

$\star \star$ Research partially supported by the Air Force Office of Scientific Research

$\star \star \star$ The paper was written while Dr. Alligood was visiting Michigan State University and the University of Maryland

Present address: George Mason University, Fairfax, VA 22030, USA
} 

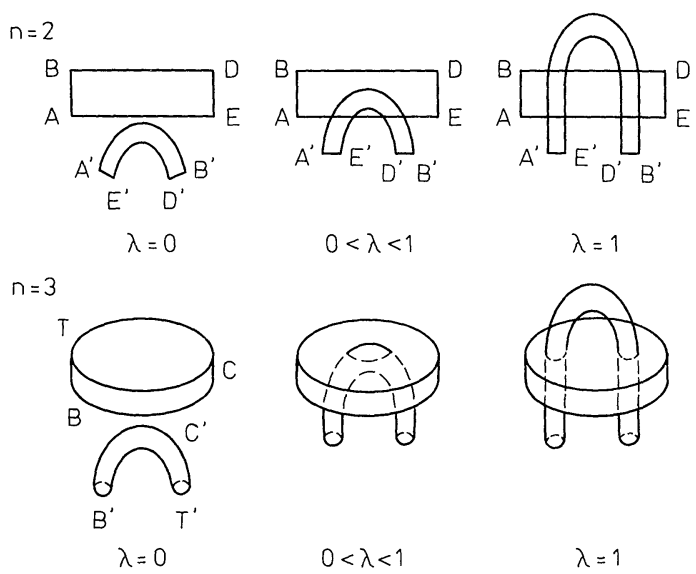

Fig. 1a-f. The typical way that a horseshoe is formed is shown in dimensions 2 and 3. The map depends on a parameter $\lambda$. For $n=2$, rectangle $A B D E$ is mapped to $A^{\prime} B^{\prime} D^{\prime} E^{\prime}$. At $\lambda=0, A B D E$ and $A^{\prime} B^{\prime} D^{\prime} E^{\prime}$ are disjoint; as $\lambda$ increases to 1 , the image $A^{\prime} B^{\prime} D^{\prime} E^{\prime}$ is pulled through $A B D E$ in the horseshoe shape indicated. For $n=3$, cylinder $C$ is mapped analogously to $C^{\prime}$, with the top $T$ and bottom $B$ mapped to $T^{\prime}$ and $B^{\prime}$, respectively. The properties required in the theorems are topological, so distortions of these pictures are allowed provided the hypotheses of the theorems are satisfied. We require that as $\lambda$ goes from 0 to 1 the images of the top and bottom (when $n=2$ ) never touch the rectangle $A B C D$, and the images of the sides never touch the sides

the map is area contracting. Then the way periodic orbits normally arise as $\lambda$ increases is through saddle-node bifurcations: a pair of orbits, both of minimum period $k$, pop into existence. One orbit is attracting and the other is unstable. (Repelling orbits are ruled out by area contraction.)

The unstable orbit will be called a saddle. Two orbits have been created, but the attractor is not altogether welcome since there are none at $\lambda=1$. What is most likely to happen is that the attractor becomes unstable through a period-doubling bifurcation. Now there are generally three orbits: 2 unstable orbits of period $k$, and an attractor of period $2 k$, though an "inverted" period-doubling bifurcation could yield an unstable orbit. Figure $2 \mathrm{a}$ shows the most common pattern, in which the period $2 k$ attractor becomes unstable as $\lambda$ increases, through period doubling. Each period doubling produces an attracting orbit of twice the previous period. A schematic diagram of the pattern in Fig. 2a in which each point represents an entire periodic orbit is shown in Fig. 2b. A more complicated pattern is shown (schematically) in Fig. 2c. In each case there is a connected network of orbits in $(x, \lambda)$ space that we refer to as a component. In both components shown there are attracting orbits of all the periods $k, 2 k, 4 k, \ldots$. Hence there is a period-doubling "cascade" of attractors. Such phenomena were first described in 1962 by Myrberg $[\mathrm{M}]$ for 1-dimensional quadratic maps.

In describing our results, it is important to distinguish two types of unstable orbits since they arise in different ways. Roughly half the unstable period $k$ orbits will be called "saddles." These arise paired with attractors of period $k$. The other unstable orbits of period $k$ are called "Mobius orbits," and these arise through 


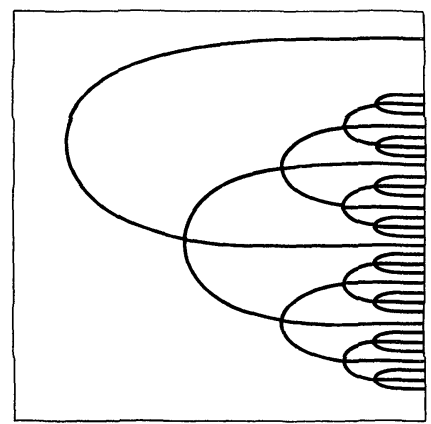

a

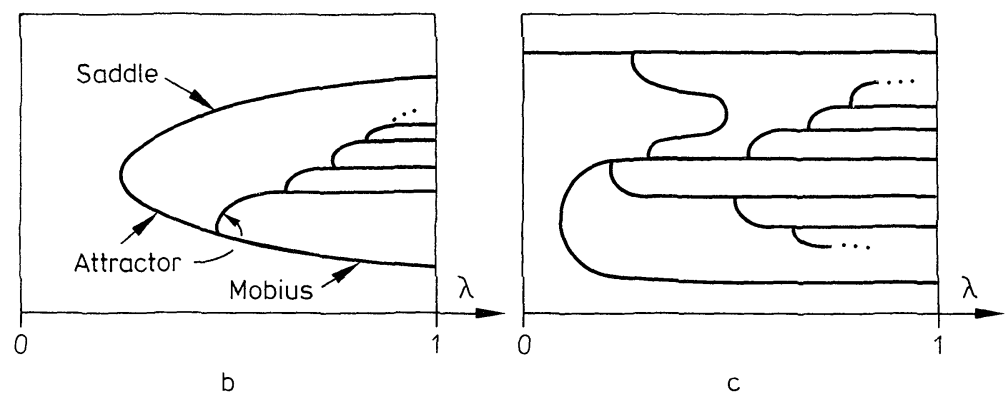

Fig. 2a-c. Diagrams of possible orbit components are shown for typical maps on $C \times I$. The same configuration is shown in $\mathbf{a}$ and $\mathbf{b}$. In $\mathbf{a}$ the points on the orbit are shown while in $\mathbf{b}$ the diagram is more schematic. The vertical axis is the space of orbits, so each point represents an entire orbit for a given $\lambda$. The same representation is used in $\mathbf{c}$ where a more complicated web of bifurcations is illustrated. The dots indicate an infinite sequency of period-doublings

period doubling: an attractor of period $k$ becomes unstable, and the unstable period $k$ orbit is a Mobius orbit. ${ }^{1}$

The general result we prove in Sect. 4 may be stated loosely as follows. Choose any saddle in the horseshoe $(\lambda=1)$. Let $k$ denote its period, (i.e., its minimum period), so the orbit consists of exactly $k$ points. Examine its component: the network of orbits connected to it, as illustrated in Fig. 2. Then, necessarily, the component will contain attractors of minimum period $k, 2 k, 4 k, \ldots$, (Theorem A, Sect. 4). That is, there will be a cascade of period doubling attractors connected to the saddle. All these must occur before the horseshoe is fully developed at $\lambda=1$. Hence the title's claim that a cascade of period-doubling attractors is a prerequisite

1 For an unstable orbit of period $k$, the Jacobian of the $k^{\text {th }}$ iterate of the map has an eigenvalue $\mu$ with $|\mu|>1$. When $\mu>1$, the orbit is a saddle. When $\mu<-1$, it is called a "Mobius" orbit reflecting the half twist indicated by a negative eigenvalue. The odd name comes from cases where a flow is being examined, and the map is a Poincare return map. Then $\mu<-1$ implies that the unstable manifold of the periodic orbit has a half twist; that is, it is a Mobius strip 
Table 1. The number of $S(N)$ of saddle orbits of minimum period $N$ in the standard horseshoe is approximately $2^{N-1} / N$

\begin{tabular}{lllrrr}
\hline $\begin{array}{l}\text { Minimum } \\
\text { period } \\
N\end{array}$ & $\begin{array}{l}\text { No. saddle } \\
\text { orbits } \\
S(N)\end{array}$ & $N$ & $S(N)$ & $N$ & $S(N)$ \\
\hline 1 & 1 & 11 &, 93 & 21 & 49,929 \\
2 & 0 & 12 & 165 & 22 & 95,232 \\
3 & 1 & 13 & 315 & 23 & 182,361 \\
4 & 1 & 14 & 576 & 24 & 349,350 \\
5 & 3 & 15 & 1091 & 25 & 671,088 \\
6 & 4 & 16 & 2032 & 26 & $1,290,240$ \\
7 & 9 & 17 & 3855 & 27 & $2,485,504$ \\
8 & 14 & 18 & 7252 & 28 & $4,792,905$ \\
9 & 28 & 19 & 13,797 & 29 & $9,256,395$ \\
10 & 48 & 20 & 26,163 & 30 & $17,894,588$ \\
\hline
\end{tabular}

for the horseshoe. This cascade is connected to the saddle of period $k$ at $\lambda=1 .^{2}$ Table 1 gives exact calculations that illustrate that there are approximately $2^{k-1} / k$ saddle orbits of (minimum) period $k$ in the horseshoe. Theorem C (Sect. 4) says that each has its own cascade. Hence there are three cascades of period 5 and almost 18 million cascades starting from period 30 orbits. Each of the latter will contain attracting orbits of period $30,60,120, \ldots$.

One part of our result had been proved by Robinson [R] (in an investigation of Newhouse's theory of horseshoes): namely, as a horseshoe is formed for an area contracting map, an attracting fixed point must be created. He also showed that for generic maps, i.e. maps whose bifurcations are not degenerate, attracting orbits of all periods must occur. Sect. 4 shows how to forego genericity hypotheses. Our main results were announced in [YA], and the announcement motivated Franks [F] to give different proofs of our results together with new related results.

The ideas in this paper are developed further in [A] where it is shown that even the Mobius orbits at $\lambda=1$ are connected to cascades. More precisely, the component of each Mobius orbit (or saddle as we prove here) of period $k$ contains a cascade of attractors of period $k, 2 k, \ldots$. It is also shown in [A] that the number of Mobius orbits of period $k$ equals the number of saddles of period $k$ plus the number of saddles of period $k / 2$ and $k / 4$, etc. Of course there are saddles of period $k / 2$, for example, only if $k$ is even.

In Sect. 2 we describe and give examples of the formation of horseshoes. Section 3 contains the development of the generic theory necessary for the main results, which are stated and proved in Sect. 4. Variations of these results are indicated in some further examples at the end of Sect. 4.

2 The main techniques in the proof are, first, to erase all the Mobius orbits in the networks illustrated in Fig. $2 b$ and c. We leave all attractors, saddles, and bifurcation orbits. Secondly we examine the orbits still connected to any given saddle at $\lambda=1$ and prove that this greatly simplified network has all the required attracting orbits of the cascade. This proof uses an orbit index developed in earlier papers. Non-generic situations in which an unusually large number of branches emanate from a bifurcation orbit are handled separately in Sect. 4 


\section{Forming Horseshoes}

Let

$$
\begin{aligned}
& C=\left\{\left(x_{1}, \ldots, x_{n}\right) \in R^{n}: x_{1}^{2}+\ldots+x_{n-1}^{2} \leqq 1 \text { and } 0 \leqq x_{n} \leqq 1\right\}, \\
& E=\left\{\left(x_{1}, \ldots, x_{n}\right) \in C: x_{1}^{2}+\ldots+x_{n-1}^{2}=1\right\}, \\
& T=\left\{\left(x_{1}, \ldots, x_{n}\right) \in C: x_{n}=1\right\}, \text { and } \\
& B=\left\{\left(x_{1}, \ldots, x_{n}\right) \in C: x_{n}=0\right\} .
\end{aligned}
$$

We consider a $C^{1}$ map $f: C \times[0,1] \rightarrow R^{n}$ (occasionally writing $f_{\lambda}$ for $f(\cdot, \lambda)$ ) such that $f_{0}(C)$ is disjoint from $C$, and, as $\lambda$ increases to $1, f_{\lambda}$ becomes a horseshoe map in a regular fashion (see Fig. 1). Specifically, writing $C_{\lambda}$ for $f_{\lambda}(C)$ and using the corresponding notation for $E, T$, and $B$, we assume the following conditions that describe how the horseshoe is formed:

(H 1) All periodic orbits in $C_{0} \cap C$ are attractors.

In particular, $C_{0} \cap C$ may be empty, as in Fig. 1.

(H 2) $T_{\lambda} \cap C, B_{\lambda} \cap C$, and $C_{\lambda} \cap E$ are empty, for all $\lambda$.

For more general examples in which horseshoes are not formed but to which the theorems of Sect. 4 apply (e.g., the examples at the end of Sect. 4), we add that the proofs of these theorems require only the following broader hypothesis, in place of $(\mathrm{H} 2)$ that no periodic orbits of $C$ touch the boundary of $C$, or more precisely:

$\left(\mathrm{H} 2^{\prime}\right)$ Every periodic orbit of $f$ that lies wholly in the closed set $C$ actually lies in the interior of $C$, when $0<\lambda<1$.

Now we describe the types of periodic orbits allowed in $C$ at $\lambda=1$ :

(H3) If $f^{k}(p, 1)=p$, for some $k \geqq 1$ and $p \in C$, then $D_{p} f^{k}(p, 1)$ has exactly one eigenvalue $\mu$ such that $|\mu|>1$ and the remaining eigenvalues satisfy $|\mu|<1$.

In terminology we introduce later, this may be restated: each periodic orbit in $C$ at $\lambda=1$ is either a saddle or Mobius orbit.

In addition, we wish to assume that the mapping is locally expansive in at most one direction. We consider two distinct cases. Whenever $f^{k}(p, \lambda)=p$ for some $\lambda \in[0,1], k \geqq 1$, and $p \in C$, and if $\mu_{1}, \ldots, \mu_{n}$ are the eigenvalues of $D_{p} f^{k}(p, \lambda)$, we assume either

(AC) (Area Contraction Hypothesis). There is a $\theta<1$ such that $\left|\mu_{i} \mu_{j}\right|<\theta$, for $i$ $\neq j, 1 \leqq i, j \leqq n$; or

(AP) (Area Preservation Hypothesis). $n=2$ and $\operatorname{det} D_{p} f(p, \lambda)=1$, (i.e., $f_{\lambda}$ preserves area, for all $\lambda$ ).

We call (AC) the area contraction hypothesis because it is satisfied for maps $F$ on $R^{n}$ if $D_{x} F$ contracts areas on every two dimensional subspace.

Let

$$
P=\left\{(p, \lambda) \in C \times I: f^{k}(p, \lambda)=p, \text { for some } k \geqq 1\right\} .
$$

We identify points $(p, \lambda)$ and $(q, \lambda)$ in $P$ if they are on the same orbit, i.e., if $f^{m}(p, \lambda)=q$, for some $m$. Now let $\mathcal{O}(f)$ be the "orbit space" - that is, the $m$ points of an orbit are represented as a single orbit in $\mathcal{O}(f)$. (See Fig. $2 \mathrm{~b}$ and $\mathrm{c}$ for schematic diagrams of orbit components.) Let $\mathcal{O}(f, k)$ be the set of orbits of $f$ of minimum period $k$. Hence $\mathcal{O}(f)=\bigcup_{k \in N} \mathcal{O}(f, k)$. For ease of notation, we sometimes write 


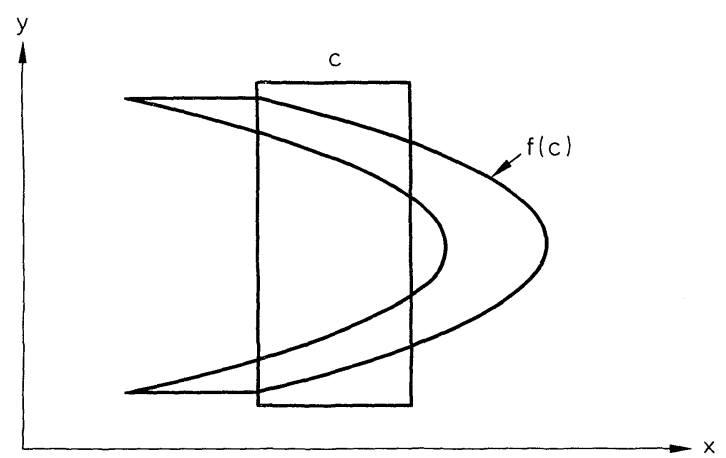

Fig. 3. The horseshoe of the Henon map is shown (see Example 1, Sect. 2)

$(p, \lambda) \in \mathcal{O}(f)$ when $(p, \lambda)$ is a point in an orbit. For a point $(p, \lambda) \in P$, let $(p, \lambda)$.

$\Lambda(p, \lambda)$ be the set of eigenvalues of $D_{p} f^{k}(p, \lambda)$, where $k$ is the minimum period of

We call $\Lambda(p, \lambda)$ the eigenvalues of $(p, \lambda)$.

Under assumption (AC), each orbit $(p, \lambda)$ in $\mathcal{O}(f)$ is in one of the following five disjoint subsets, classified according to the location of the eigenvalues of the orbits:

(1) the set $A$ of attracting orbits, $(|\mu|<1$, for all $\mu$ in $\Lambda(p, \lambda))$;

(2) the set $M$ of Mobius orbits, $(\mu<-1$, for some $\mu$ in $\Lambda(p, \lambda))$;

(3) the set $S$ of saddle orbits, $(\mu>+1$, for some $\mu$ in $\Lambda(p, \lambda))$;

(4) $B_{+}=$the set of orbits having +1 as an eigenvalue; and

(5) $B_{-}=$the set of orbits having -1 as an eigenvalue.

The reader should note our distinction between $S$ and $M$. In particular, even though orbits in $M$ have a saddle structure, we distinguish them from orbits in $S$ because of the eigenvalue $\mu$ in $(-\infty,-1)$. Notice also that in each of cases (2)-(5), at most one eigenvalue in $\Lambda(p, \lambda)$ can lie on or outside the unit circle in the complex plane. We sometimes specify the map for clarity by writing $A(f)$ or $B_{+}(f)$, etc.

Under assumption (AP), the set $A$ is replaced by the set $L$ of elliptic orbits, $(|\mu|=1$ and $\mu \neq 1$ or -1 , for both $\mu$ in $\Lambda(p, \lambda))$. In this case the complex conjugate of an eigenvalue is always an eigenvalue.

We conclude this section with three examples of functions which satisfy $(\mathrm{H} 1)-(\mathrm{H} 3)$ and (AC) or (AP), and hence to which the theorems of Sect. 4 apply.

Example 1. For $(x, y) \in R^{2}$, the Henon map, given by

$$
f(x, y)=\left(\lambda-x^{2}+B y, x\right)
$$

( $\lambda$ and $B$, real constants), is area preserving for $B=-1$ and area contracting for $0 \leqq|B|<1$. Fixing $B$ and letting $\lambda$ vary, Devaney and Nitecki [DN] proved that, in the cases $-1 \leqq B<0$ (orientation preserving), $f$ forms a horseshoe [in particular, $f$ satisfies ( $\mathrm{H} 1)-(\mathrm{H} 3)$ on some subset of $\left.R^{2}\right]$. The horseshoe configuration is shown in Fig. 3. Notice that as $\lambda$ is decreased in Eq. (2.1), the image $f(C)$ is translated to the left without any other change in size or shape. Hence for $\lambda$ sufficiently negative, $C$ and $f(C)$ are disjoint. As $\lambda$ is increased from this value, the horseshoe is formed. 


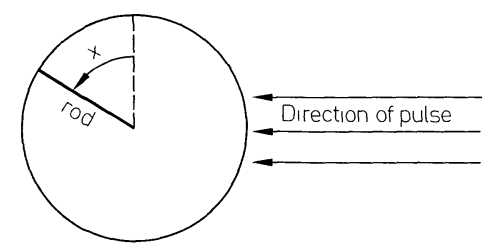

Fig. 4. The rotor of Example 2 (Sect. 2) is shown. The revolving rod is kicked periodically (at times $t=0,1,2, \ldots)$ from right to left

In particular, Devaney and Nitecki show that for $\lambda<-(1+|B|)^{2} / 4, f$ has no periodic points, and for $\lambda>(5+2 \sqrt{5})(1+|B|)^{2} / 4$, a horseshoe exists [i.e., (H 3$)$ is satisfied].

Setting $B=0$ and neglecting the second coordinate (which is then redundant), we obtain the one-dimensional quadratic map

$$
g(x)=\lambda-x^{2} .
$$

For $\lambda<-\frac{1}{4}, g$ has no periodic points; for $\lambda \geqq 2, g$ has unstable periodic points of every period. (When $\lambda=2$, the interval $[-2,2]$ maps onto itself with $g(-2)$ $=g(2)=-2$. It can then be shown that $g^{n}$ has $2^{n}$ fixed points in $[-2,2]-$ half of which have positive derivative, for each $n \geqq 0$.)

In the Henon example above, the map (2.1) can be written as a dynamical process:

$$
x_{n+1}=\lambda-x_{n}^{2}+B x_{n-1} .
$$

The analysis of [DN] showing that a horseshoe is created can be applied with little change to

$$
x_{n+1}=\lambda-x_{n}^{2} \pm B x_{n-k}
$$

with $0 \leqq \lambda<1$ - or, equivalently, letting $\beta=B^{1 /(k+1)}$, to the $k+1$ dimensional map,

$$
f\left(x, y_{1}, \ldots, y_{k}\right)=\left(\lambda-x^{2} \pm \beta y_{k}, x, \beta y_{1}, \ldots, \beta y_{k-1}\right) \text {. }
$$

For $\beta=0, D f$ is a matrix with one dimensional range; hence, for any bounded set $C$ $\times[0,1]$ in $R^{k+1} \times R$, there will be a $\beta_{0}$ for which (AC) is satisfied when $\beta<\beta_{0}$.

Example 2. In this example, we look at the equations of motion of a rotor driven by a periodic force. In particular, consider a one unit long rigid rod rotating in a plane (see Fig. 4) which is kicked (from a fixed direction) at regular times $(t=0,1,2, \ldots)$. Then letting

$$
\begin{aligned}
x_{n} & =\text { angle of the rod at time } n, \\
y_{n} & =\text { distance travelled from } n \text { to } n+1, \\
k & =\text { frictional constant, and } \\
P & =\text { magnitude of the kick, }
\end{aligned}
$$


we have

$$
x_{n+1}=\left(x_{n}+y_{n}\right) \bmod 2 \pi, \quad y_{n+1}=\alpha y_{n}-\lambda \cos \left(x_{n}+y_{n}\right),
$$

where $\alpha=e^{-k}$ and $\lambda=P\left(\frac{1-e^{-k}}{k}\right)$.

The system (2.4) is a variant of the standard map of Chirikov [C] in that dissipation is considered (when $\alpha<1$ ). A more general derivation of a perturbed non-linear oscillator (which is equivalent to (2.4) under a change of variables) is given by Zaslovsky [Z]. In [H], Holmes uses this system to model the motion of a ball bouncing vertically on a sinusoidally vibrating plate (with certain approximating assumptions). In this paper, he shows that in both the area preserving case $(\alpha=1)$ and the area contracting case $(0<\alpha<1)$ a horseshoe is formed as $\lambda$ increases from 0 . Specifically, when $\alpha=1$, a horseshoe exists for $\lambda \geqq 5 \pi$. (When $\alpha<1$, the value of $\lambda$ for which a horseshoe first exists is not specified, but varies with $\alpha$ and approaches $5 \pi$ as $\alpha \rightarrow 1$.) We should mention that since (2.4) has fixed points in $R^{2}$ for all $\lambda$, the domain considered for horseshoe formation can be restricted to satisfy $(\mathrm{H} 1)$ and (H 2). Numerical studies of chaotic behavior for (2.4) as the horseshoe is formed are reported in [SY].

Example 3. Let $f_{\lambda}$ be a one-parameter family of homeomorphisms on $R^{n}$, and let $p$ be a fixed point of $f_{\lambda}$, for all $\lambda$. We assume that $p$ has one eigenvalue $\mu_{1}$ such that $\left|\mu_{1}\right|>1$; all other eigenvalues satisfy $\left|\mu_{j}\right|<1$, and $\left|\mu_{1} \mu_{j}\right|<1, j=2, \ldots, n$. Suppose further that a non-degenerate tangency is formed between the stable and unstable manifolds of $p$ as $\lambda$ varies. Then for some range of parameter values near that at which tangency occurs (either before or after), and for $k$ sufficiently large, the $k^{\text {th }}$ iterate of the map will form a horseshoe. I.e., $(\mathrm{H} 1)-(\mathrm{H} 3)$ will be satisfied on some subset $C$ of $R^{n}$ near the point of tangency (see $[\mathrm{S}, \mathrm{N}, \mathrm{R}, \mathrm{GH}]$ ).

\section{The Orbit Index and Oriented Paths of Orbits}

Each saddle, attractor, or Mobius orbit in $C \times[0,1]$ persists as the parameter is varied, so it lies on a segment of orbits having the same period. All the orbits on the segment are saddles or all are attractors or all are Mobius orbits. Each segment is monotonic in $\lambda$, that is it has at most one orbit for each $\lambda$. Each segment ends in a bifurcation orbit (i.e. an orbit in $B_{+}$or $B_{-}$), except when the segment ends at $\lambda=0$ or $\lambda=1$. When it extends to $\lambda=0$, it is a segment of attractors terminating in an attractor at $\lambda=0$ (since by $H 1$, the only orbits allowed at $\lambda=0$ are attractors). Similarly, when it ends at $\lambda=1$, it is a segment of saddles ending with a saddle at $\lambda=1$, or a segment of Mobius orbits ending with a Mobius orbit at $\lambda=1$.

In general, the structure of orbit components at bifurcation points can be quite complicated. However, this structure is particularly nice for a set $K$ of maps $g(x, \lambda)$ studied in [AY]. We will say $g$ is generic when $g$ is in $K$. A map $g$ is in $K$ if all its bifurcation orbits are of the two simple types described below. Furthermore, $g$ has only a finite number of bifurcation orbits of any given period in a specified compact set. The value of studying the set $K$ derives from the fact that it is dense in the space of all $C^{1}$ maps $f(x, \lambda)$; that is, $f$ and its partials can be uniformly approximated on $C \times[0,1]$ by maps $g \in K$. The cascade properties of $f$ can be inferred from the 


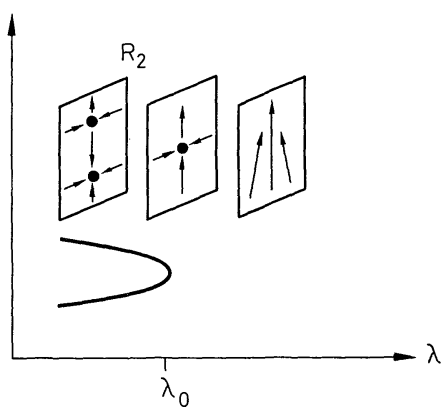

a

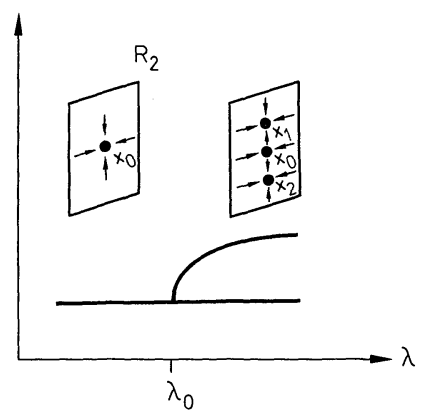

b

Fig. 5a and b. The two generic bifurcations are shown schematically. The curve in a depicts a saddle-node bifurcation at $\lambda=\lambda_{0}$ : each point on the curve represents a periodic orbit [i.e., a point in $\mathcal{O}(g)]$. The three cross-sectional pictures show an example in $R^{2}$ of two fixed points (an attractor and a saddle) which coalesce in a saddle node and disappear as $\lambda$ increases. The branched curve in b depicts a period-doubling bifurcation at $\lambda=\lambda_{0}$ : points on the upper branch represent orbits with twice the period of those on the lower branches. In $\mathbf{b}$, the first cross-sectional picture (for $\lambda<\lambda_{0}$ ) shows an example in $R^{2}$ of an attracting fixed point $x_{0}$ approaching a period-doubling bifurcation. At $\lambda=\lambda_{0}$, a change in the stability of $x_{0}$ occurs, and $x_{0}$ becomes Mobius. The second crosssectional picture (for $\lambda>\lambda_{0}$ ) shows the Mobius fixed point $x_{0}$ and the period two attractor (points $x_{1}$ and $x_{2}$ ) which has bifurcated from the $x_{0}$-path at $\lambda=\lambda_{0}$. Since $x_{1}$ and $x_{2}$ are on the same orbit, they are represented by a single point on the upper branch of the schematic graph

generic situation. The set $K$ is discussed in detail in [AY]. A somewhat technical proof that $K$ is $C^{1}$ dense is given in the Appendix to [AMY]. Here we describe the types of bifurcation orbits that are allowed for $g \in K$ when $g$ satisfies the area contraction hypothesis (AC):

(1) Orbits in $B_{+}(g)$ are "saddle-node" bifurcation orbits from which two segments of non-Mobius orbits emanate in $(x, \lambda)$-space: the orbits on one are saddles, and orbits on the other are attractors (see Fig. 5a). Passing through the bifurcation orbit, from the segment of saddles to the segment of attractors one eigenvalue crosses +1 (with non-zero derivative). ${ }^{3}$ Near the saddle-node, orbits on both segments have the same minimum period as the bifurcation orbit.

(2) Orbits in $B_{-}(g)$ are "period-doubling" bifurcation orbits from which three segments of orbits - two non-Mobius and one Mobius - emanate (see Fig. 5b.) Orbits on one of the non-Mobius segments have twice the minimum period of the bifurcation orbit, while orbits on the other two segments have the same minimum period. Following the low-period path of orbits through the bifurcation orbit, one eigenvalue crosses -1 (with non-zero derivative). ${ }^{3}$

For generic maps, the topological structure of a connected set of orbits can be a complicated network or graph with two segments coming from each $B_{+}$orbit and three segments coming from each orbit in $B_{-}$; but the structure of non-Mobius orbits is quite simple, since by not considering Mobius orbits we eliminate exactly

3 These conditions are special cases of the transversality conditions mentioned in the Appendix to $[\mathrm{AMY}]$ 


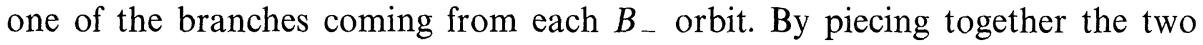
segments of non-Mobius orbits, at each bifurcation orbit, we construct a (onedimensional) path. Proceeding from bifurcation orbit to bifurcation orbit, and piecing together successive segments we construct a maximal path. Notice that each hyperbolic non-Mobius orbit lies on a unique (fully extended) path of orbits. This path can be a closed loop in some cases. In other cases it leads us through an infinite sequence of bifurcation orbits. Of course then the path would never pass twice through the same orbit, and the periods of the bifurcation orbits would be unbounded. A fully extended path of non-Mobius orbits will be called a snake. Hence we have the following lemma:

\section{Lemma 3.1. Let $g \in K$. Each non-Mobius orbit lies in a snake.}

We remark that while the snake $\Gamma$ may have an endpoint in $\operatorname{bdry}(C \times I)$, i.e., the boundary of $C \times I$, we will show in Lemma 3.3 that $\Gamma$ intersects bdry $(C \times I)$ in at most one point (which must be a saddle at $\lambda=1$ or an attractor at $\lambda=0$ ).

The main tool used in proving the results in this paper is the $\phi$-index (also called the orbit index). It was introduced in [MY, CMY, AMY, AY2], for studying the global continuability of periodic orbits of ordinary differential equations, often specifically the family of orbits that appears in a Hopf bifurcation. The paper applies the orbit index to a rather different class of problems - maps whose periodic orbits have at most one eigenvalue outside the unit circle. Recall that for a $C^{1}$ map $g: R^{n} \rightarrow R^{n}$ with a periodic point $p$ of minimum period $k$, the term "eigenvalue of $p$ " means an eigenvalue of $D_{x} g^{k}(p)$. For the moment $\lambda$ plays no role so it is not mentioned.

The orbit index is simplest to define for hyperbolic orbits, (i.e., orbits that have no eigenvalues with absolute value 1), and there is no need here to consider the index for more general orbits. In this paper the hyperbolic orbits studied have at most one eigenvalue outside the unit circle, and for such orbits the orbit index is

$$
\begin{aligned}
& \phi=0 \text { for Mobius orbits } \\
& \phi=1 \text { for attractors } \\
& \phi=-1 \text { for saddles. }
\end{aligned}
$$

For hyperbolic orbits in general, Mobius orbits are defined to be orbits for which the number $\sigma_{-}$(counted with multiplicities) of real eigenvalues less than -1 is odd. For these orbits, $\phi$ is always 0 . For hyperbolic orbits that are not Mobius orbits, (i.e., $\sigma_{-}$is even), there are two cases:

$\phi=1$ if the number $\sigma_{+}$of real eigenvalues greater than +1 is even;

$\phi=-1$ if $\sigma_{+}$is odd.

For a component (i.e. a maximal connected subset) $S$ of $\mathcal{O}(g)$, let $S_{\lambda}$ be the orbits in $S$ that occur at a specified $\lambda$. The proof of the following invariance property is in [MY] for generic maps and can be obtained by analyzing the stability of orbits and the direction of their paths in neighborhoods of the bifurcation orbits. This property can be generalized greatly, but it is sufficient here to assume the map is generic. 


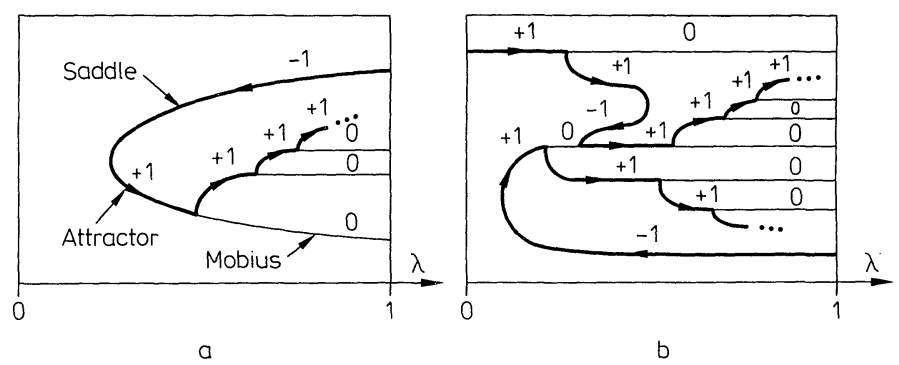

Fig. 6a and b. Two schematic diagrams of orbit components for a generic map on $C \times I$ are shown. Each point on the branched curves represents a periodic orbit; the generic bifurcations are drawn as in Fig. 5. The thicker paths represent snakes (i.e., paths of non-Mobius orbits); the thinner ones, paths of Mobius orbits. In addition, the orbit index of orbits on each branch is indicated, as is the orientation of the snakes. In $\mathbf{a}$, one snake is shown which begins with a saddle orbit at $\lambda=1$, then changes $\lambda$-direction at the saddle node, and ends in a cascade of attractors. A more complicated web of bifurcations is shown in $\mathbf{b}$. Here one component of orbits contains two snakes. One begins with an attractor at $\lambda=0$; the other, with a saddle at $\lambda=1$. Both snakes end in cascades of attractors

Proposition 3.2. If the periods of orbits in $S$ are bounded, then, for each $\lambda, S_{\lambda}$ contains at most a finite number of orbits, and

$$
\Phi(\lambda)=\sum_{p \in S_{\lambda}} \phi(p)
$$

is constant; that is, it does not depend on $\lambda$.

This result is applied by examining $\lambda$ just before a bifurcation and again for $\lambda$ just after the bifurcation.

The invariance property of $\phi$ (Proposition 3.2) allows us to choose a preferred direction on a snake $\Gamma$. Notice that $\Gamma$ changes direction (from $\lambda$ increasing to $\lambda$ decreasing, or vice versa) only at bifurcation orbits, in which case the index changes from +1 to -1 , or vice versa. For convenience, we choose the following orientation on each $\Gamma$ (see Fig. 6): let $(p, \lambda) \in \Gamma$,

(1) if $\phi(p)=+1$, follow $\Gamma$ through increasing $\lambda$ values;

(2) if $\phi(p)=-1$, follow $\Gamma$ through decreasing $\lambda$ values.

By examining the orientation of the snake through an orbit at $\lambda=0$ or $\lambda=1$, we will show in the proof of the next lemma that the snake begins at the orbit. Since it cannot have two beginning ends, only one end can be in the boundary.

Lemma 3.3. Let $g \in K$ satisfy $(\mathrm{H} 1)-(\mathrm{H} 3)$. Each snake $\Gamma$ of (non-Mobius) orbits in $\mathcal{O}(g)$ has at most one point $(p, \lambda)$ in bdry $(C \times I)$, and such a boundary point must be a saddle at $\lambda=1$ or an attractor at $\lambda=0$.

Proof. By (H 2) there are no periodic orbits in $E \times I=\operatorname{bdry}(C) \times I$. Let $\Gamma$ be a snake of $\mathcal{O}(g)$, and suppose that $(p, 1) \in \Gamma$. Then, $\phi(p) \neq 0$, and by $(\mathrm{H} 3)$ it is a saddle, $\phi(p)=-1$, and we follow $\Gamma$ through decreasing $\lambda$ values from $(p, 1)$, as its orientation requires. Hence the path leads into $C \times I$, and $(p, 1)$ is the beginning of the oriented path. Similarly if $(p, 0) \in \Gamma$, then $p$ is an attractor by $(\mathrm{H} 1)$ and $\phi=+1$, so 
we follow the path with $\lambda$ increasing. Hence the path again leads into $C \times I$. Hence any point of $\Gamma$ at $\lambda=0$ or $\lambda=1$ is necessarily the beginning of the oriented curve and cannot be the final end.

Lemma 3.4. Let $g \in K$ satisfy (AC), and let $(p, \lambda)$ be a period-doubling bifurcation orbit (i.e., $\left.(p, \lambda) \in B_{-}(g)\right)$. The low period segment of non-Mobius orbits emanating from $(p, \lambda)$ consists of attractors.

Proof. Suppose otherwise - that the segment consists of saddles. Then along this segment each orbit has an eigenvalue in $(1, \infty)$. However, following the segment into $(p, \lambda)$, another eigenvalue must approach -1 . The bifurcation orbit would have -1 as an eigenvalue and would have another in $[1, \infty)$ contradicting (AC).

The following proposition shows (for $g \in K$ ) how components of orbits which originate in the horseshoe must terminate:

Proposition 3.5. Let $g \in K$ satisfy $(\mathrm{H} 1)-(\mathrm{H} 3)$ and $(\mathrm{AC})$, and let $\Gamma$ be the snake containing a saddle orbit $(q, 1)$ of period $k$. Then the (minimum) periods of orbits in $\Gamma$ are unbounded. Furthermore, there is an attractor of (minimum) period $2^{n} k$ on $\Gamma$, for each $n \geqq 0$.

Proof. A snake may form a closed loop. When that is not the case, the path can never pass through the same orbit twice. The snake $\Gamma$ begins on the boundary so it cannot be a loop. Since snakes are constructed segment by segment, leading us from bifurcation orbit to bifurcation orbit, $\Gamma$ must pass through an infinite number of bifurcation orbits: $\Gamma$ has a beginning (at $\lambda=1$ ), but it has no end. Hence, the periods along $\Gamma$ must be unbounded, since for $g \in K$ there are only finitely many bifurcation orbits of each period. In fact there must be a last bifurcation orbit of period less than $k$, so the periods tend to $\infty$ as we follow the path. In particular, $\Gamma$ must pass through $B_{-}$bifurcation orbits of period $k, 2 k, 4 k, \ldots$ By Lemma $3.4, \Gamma$ has segments of attractors of period $2^{n} k$, for each $n \geqq 0$.

With virtually no change in techniques we also obtain:

Proposition 3.6. For $g$ as in Proposition 3.5, let $\Gamma$ be a snake containing an attractor at $\lambda=0$. Then again there are attractors in $\Gamma$ of minimum period $2^{n} k$, for each $n \geqq 0$.

\section{Main Results}

In this section, we use the results of Sect. 3 for generic maps and limit arguments to prove the following theorems, dropping genericity assumptions.

Theorem A. Let $f$ satisfy $(\mathrm{H} 1)-(\mathrm{H} 3)$ and the area contracting hypothesis $(\mathrm{AC})$. For any saddle orbit $(p, 1)$ or attractor $(p, 0)$ in $\mathcal{O}(f)$ of minimum period $k$, the component of $\mathcal{O}(f)$ containing $(p, 1)$ or $(p, 0)$ has attracting periodic points whose (minimum) period is $2^{m} k$, for each $m \geqq 0$. 
Theorem B. Let $f$ satisfy $(\mathrm{H} 1)-(\mathrm{H} 3)$ and the area preserving hypothesis (AP) $(n=2)$. If $f$ is orientation preserving, then for any saddle orbit $(p, 1) \in \mathcal{O}(f)$ of minimum period $k$, the component of $\mathcal{O}(f)$ containing $(p, 1)$ has elliptic periodic points whose (minimum) period is $2^{m} k$, for each $m \geqq 0$.

We define a cascade from period $k$ to be a sequence $\left\{Q_{0}, Q_{1}, \ldots\right\}$ of (non-empty) components of $A$ such that, for each $m \geqq 0$, the orbits in $Q_{m}$ have period $2^{m} k$, and $Q_{0} \cup Q_{1} \cup \ldots$ is contained in one component of $\mathscr{O}(f)$. When there are an infinite number of saddle orbits at $\lambda=1$, such as in the horseshoe map (see, for example $[\mathrm{S}])$, the following theorem says that an infinite number of disjoint cascades must exist. (See also Table 1, which shows the number of saddles in the horseshoe up through minimum period 30.) Two cascades, $\left\{Q_{m}\right\}$ and $\left\{R_{m}\right\}$, are disjoint when there are no periodic orbits that are in both cascades (i.e., $Q_{0} \cup Q_{1} \cup \ldots$ is disjoint from $\left.R_{0} \cup R_{1} \cup \ldots\right)$. Notice that this definition does not preclude two disjoint cascades from being in the same component of $\mathcal{O}(f)$.

Theorem C. Let $f$ satisfy $(\mathrm{H} 1)-(\mathrm{H} 3)$ and (AC), and let $N$ be the number of saddle orbits of (minimum) period $k$ in $C \times\{1\}$ plus the number of attractors of period $k$ in $C \times\{0\}$. Then there are at least $N$ disjoint cascades from period $k$ in $\mathcal{O}(f)$.

When $f$ is generic, these results follow from the lemmas of Sect. 3. We now develop the general case. For the proof of each theorem, we consider a sequence $g_{0}, g_{1}, \ldots$ of functions in $K$ such that $\lim _{i \rightarrow \infty} g_{i}=f$ in the $C^{1}$ topology (uniform convergence of $g_{i}$ and $D g_{i}$ on $C \times I$ to $f$ and $D f$, respectively). If $\left(p_{i}, \lambda_{i}\right)$ is in $\mathcal{O}\left(g_{i}, k\right)$ and if $\lim \left(p_{i}, \lambda_{i}\right)=(q, \lambda)$, then $(q, \lambda)$ is a periodic point of $f$, and $k$ is a multiple of the period $j$ of $(q, \lambda)$. Let $m=k / j$.

Lemma 4.1. If $m>1$, then 1 is an eigenvalue of $D_{q} f^{k}(q, \lambda)$. In addition, for each $i$, there exists an eigenvalue $\mu_{i}$ of $D_{p} g_{i}^{k}\left(p_{i}, \lambda_{i}\right)$ such that $\lim _{i \rightarrow \infty} \mu_{i}=1$.

Proof of Lemma. Suppose 1 is not an eigenvalue of $D_{q} f^{k}(q, \lambda)$. Then 0 is not an eigenvalue of $D_{q}\left[f^{k}(q, \lambda)-q\right]$. By the Implicit Function Theorem, there is a path (in $\lambda$ ) of roots of $f^{k}(q, \lambda)-q=0$ through $(q, \lambda)$; hence there is a path of fixed points of $f^{k}$ through $(q, \lambda)$. Furthermore, given a sufficiently small $\varepsilon$-neighborhood $W$ of $(q, \lambda)$, there exists a $\delta>0$ such that if $\left\|g-f^{k}\right\|_{C^{1}}<\delta$, then the set of fixed points of $g$ in $W$ is a path (in $\lambda$ ). Choosing $i$ sufficiently large so that $\left\|g_{i}^{k}-f^{k}\right\|_{C^{1}}<\delta$ and $g_{i}^{k}$ has $m$ fixed points in $W \cap\left(C \times\left\{\lambda_{i}\right\}\right)$, we reach a contradiction. The second statement follows from the fact that eigenvalues vary continuously in the $C^{1}$ topology.

A version of the preceding lemma appears in [CMY] (the Virtual Period Proposition); in particular, it is shown that there is a divisor $r$ of $m$ for which an $r^{\text {th }}$ root of unity is an eigenvalue of $D_{q} f(q, \lambda)$.

In the following proofs, we omit the $\lambda$-coordinate in $(p, \lambda)$ when it is not relevant.

Proof of Theorem $A$. We assume that $g_{i} \in K$ satisfies (H 1)-(H 3) and (AC) and that $g_{i}(\cdot, 0)=f_{0}$ and $g_{i}(\cdot, 1)=f_{1}$, for each $i$. (The techniques of [AMY] allow such a 
perturbation ${ }^{4}$.) Let $(p, 1)$ be a saddle orbit of $f_{1}$. [The proof for $(p, 0)$ an attractor of $f_{0}$ is the same.] Then, for each $i,(p, 1)$ lies on a snake $\Gamma_{i}$ of (non-Mobius) orbits of $g_{i}$. Recall that on $\Gamma_{i}$ periods can jump discontinuously only by a factor of 2 (or $\frac{1}{2}$ ). Hence on each $\Gamma_{i}$ [followed from $\left.(p, 1)\right]$ there is (for each $m \geqq 0$ ) at least one orbit in $B_{-}\left(g_{i}\right)$ where the minimum periods jump from $2^{m} k$ to $2^{m+1} k$. Following $\Gamma_{i}$ from $(p, 1)$, let $\beta_{i, m}$ be the first such orbit. Fix $i$ and $m$, and let $G_{i}$ be the set of orbits on $\Gamma_{i}$ between $\beta_{i, m}$ and $\beta_{i, m+1}$. Parametrize $G_{i}$ by $t, 0 \leqq t \leqq 1$. Let $\mu_{j}(t)(j=1, \ldots, n)$ be the eigenvalues of $G_{i}(t)$. We assume that $\lim _{t \rightarrow 0^{+}} \mu_{1}(t)=1$ (guaranteed by Lemma 4.1) and that $\lim _{t \rightarrow 1^{-}} \mu_{s}(t)=-1$, for some $s$ (possibly $s=1$ ), since $\beta_{i, m+1}$ is in $B_{-}$. By (AC), $\left|\mu_{j}(t) \mu_{k}(t)\right|<\theta<1, j \neq k, 1 \leqq j, k \leqq n$. We claim there exist orbits on $G_{i}$ such that $\left|\mu_{j}\right| \leqq \sqrt{\theta}$, for all $j$. Suppose not. Then for each $t \in I,\left|\mu_{j}(t)\right|>\sqrt{\theta}$, for some $j$. By (AC), there is at most one such $j$. Since no other path of eigenvalues can cross the circle $|\mu|=\sqrt{\theta}$ (in the complex plane) while $\left|\mu_{1}(t)\right|>\sqrt{\theta}$, it must be the case that $\left|\mu_{1}(t)\right|>\sqrt{\theta}$ for all $t$. Then $\lim _{t \rightarrow 0^{+}} \mu_{1}(t)=1$ and $\lim _{t \rightarrow 1^{-}} \mu_{1}(t)=-1$. The fact that $\left|\mu_{1}(t)\right|>0$ implies that $\mu_{1}(t)$ cannot be real for all $t$ in $(0,1)$. Thus for some $t=t^{*}$, the complex conjugate $\overline{\mu_{1}\left(t^{*}\right)}$ is an eigenvalue of $G_{i}\left(t^{*}\right)$ distinct from $\mu_{1}\left(t^{*}\right)$, and $\left|\mu_{1}\left(t^{*}\right) \overline{\mu_{1}\left(t^{*}\right)}\right|>\theta$, a contradiction.

Let $v_{i, m}$ be an orbit of period $2^{m+1} k$ on $G_{i}$ such that $|\mu| \leqq \sqrt{\theta}$, for all eigenvalues $\mu$ of $v_{i, m}$. Taking limits as $i \rightarrow \infty,\left\{v_{i, m}\right\}_{i \in N}$ must have a subsequence which converges to an orbit $v_{m}$ of $f$. Since $|\mu| \leqq \sqrt{\theta}$, for all eigenvalues $\mu$ of $v_{m}, v_{m}$ is an attractor. In particular, since $\mu^{j} \neq 1$ for any $j>1$, by Lemma 4.1, the period of $v$ is $2^{m+1} k$. The existence of an attractor $v_{0} \in \mathcal{O}(f)$ of period $k$ is argued similarly, except that $\beta_{i, 0}$ is perhaps in $B_{+}\left(g_{i}\right)$ rather than in $B_{-}\left(g_{i}\right)$, (i.e., the period-doubling bifurcations described in the proof follow an initial saddle-node on $\Gamma_{i}$, whose period is less than or equal to $k$, by Lemma 3.4).

Now let $\Gamma_{i}^{m}$ be the segment of $\Gamma_{i}$ with endpoints $(p, 1)$ and $v_{i, m}$. Notice that all orbits on $\Gamma_{i}^{m}$ have period less than $2^{m+2} k$. Let $\Gamma^{m}=\left\{x: x=\right.$ a limit point of $\left\{x_{i}\right\}_{i \in N^{\prime}}$ where $\left.x_{i} \in \Gamma_{i}^{m}\right\}$. Then $\Gamma^{m}$ is a compact, connected set of orbits containing $v_{m}$ and $(p, 1)$. Hence we conclude that $\left\{v_{m}\right\}_{m \geqq 0}$ is in the component of $\mathcal{O}(f)$ containing $(p, 1)$.

Proof of Theorem B. Following the notation in the proof of Theorem A, on each $G_{i}, \lim _{t \rightarrow 0^{+}} \mu_{1}(t)=+1$ and $\lim _{t \rightarrow 1^{-}} \mu_{s}(t)=-1, s=1$ or 2 . [Here we do not assume either (AC) or (AP) for $g_{i} \in K$.] Since we are assuming $f$ is (AP), for $i$ sufficiently large, $\operatorname{det}\left(D g_{i}^{k}\right)=\mu_{1}(t) \mu_{2}(t)$ is approximately 1 , where $k=2^{m+1}$; in particular, $\mu_{1}(t) \mu_{2}(t)>\theta>0$. Hence $\mu_{1}(t)$ and $\mu_{2}(t)$ are approximately 1 for $t$ near 0 and are

4 The existence of local perturbations follows from [B] - i.e., in an $\varepsilon$-ball the map $f$ may be perturbed so that all orbits of period smaller than a given bound are generic. We use Peixoto's technique $[\mathrm{P}]$ for patching such local perturbations together to obtain a global one. In the Appendix to [AMY], these ideas are applied to prove that the set $K$ of maps is residual. In order for $f_{0}$ and $f_{1}$ to remain unchanged (both are in $K$ ) in the constructed perturbed map $g$, we simply let the size of the $\varepsilon$-balls go to 0 at $\lambda=0$ and $\lambda=1$. [Similarly, we may assume that $g$ equals $f$ on the boundary of $C$, when using hypothesis $\left.\left(\mathrm{H}^{\prime}\right)\right]$ 


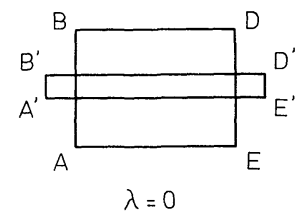

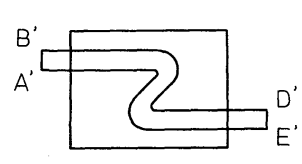

$0<\lambda>1$

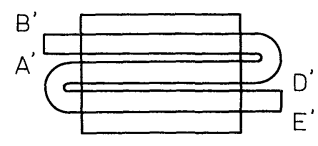

$\lambda=1$

Fig. 7. The formation of a non-standard horseshoe is shown (see Example 1, Sect. 4). At $\lambda=0$, rectangle $A B D E$ maps to rectangle $A^{\prime} B^{\prime} D^{\prime} E^{\prime}$, and there is a saddle fixed point. Throughout the construction $(0 \leqq \lambda \leqq 1)$, sides $A B$ and $D E$ map outside rectangle $A B D E$, as shown, and the image of the rectangle remains disjoint from sides $A E$ and $B D$. At $\lambda=1$, the $n^{\text {th }}$ iterate of the map has $3^{n}$ fixed points - all of which are either saddles or Möbius orbits

approximately -1 for $t$ near 1 , and must be non-real for some range of $t$. For each $\eta,-\pi<\eta<\pi$, there exists a $t$ such that $\arg \left(\mu_{s}(t)\right)= \pm \eta, s=1,2$. Taking limits (for $\eta$ $\neq 0$ ) and applying Lemma 4.1, as in the proof of Theorem A, the result is obtained.

Proof of Theorem $C$. Let $(p, 1)$ and $(r, 1)$ be two saddles of period $k$, lying on snakes $\Gamma_{p, i}$ and $\Gamma_{r, i}$ of (non-Mobius) orbits of $g_{i}$, for each $i \in N$. Notice that $\Gamma_{p, i}$ and $\Gamma_{r, i}$ are disjoint. Fix $m, m \geqq 0$, and let $A_{p, i}$ be a component of attractors on $\Gamma_{p, i}$ of period $2^{m} k$. Then $A_{p, i}$ is a path (in $\left.\lambda\right)$ of orbits whose endpoints are in $B_{+}\left(g_{i}\right)$ or $B_{-}\left(g_{i}\right)$. Let $A_{p}=\lim _{i \rightarrow \infty} A_{p, i}$ be the set of limit points of sequences $\left\{a_{i}\right\}_{i \in N}$, where $a_{i} \in A_{p, i}$. Then $A_{p}$ is a (closed) path of orbits of $f$. Let $\beta_{1}$ and $\beta_{2}$ be the endpoints of $A_{p}$, and let $Q_{p}=A_{p}-\left\{\beta_{1}, \beta_{2}\right\}$. By Lemma 4.1 and arguing as in the proof of Theorem A, orbits in $Q_{p}$ are attractors of period $2^{m} k$. (In particular, these orbits are isolated in $x$-space.) Hence $Q_{p}$ (respectively, $Q_{r}$ ) is a component of attractors. Again, by Lemma 4.1, $Q_{p}$ and $Q_{r}$ are disjoint.

Remarks. If we replace $A$ by $L$ (the set of elliptic orbits) in the definition of cascade, then Theorem $\mathrm{C}$ holds when $f$ is (AC).

In Theorem B, if $f$ is orientation reversing, the existence of an elliptic point of period $k$ is not assured by these techniques, since then +1 and -1 can simultaneously be eigenvalues of an orbit under (AC). The existence of elliptic points of period $2^{m} k$, for each $m \geqq 1$, is still obtained.

Further Examples. Minor variants of the theorems will occasionally be useful. We illustrate such variations in the following two examples:

1. In numerical studies, one occasionally sees a horseshoe formed as in Fig. 7. We assume that the map shown is area decreasing and that there is some regularity of the stretching in (i) and (iii), (i.e., all orbits at $\lambda=0$ and $\lambda=1$ are hyperbolic). Then there will be exactly one periodic orbit at $\lambda=0$, and it will be a saddle; at $\lambda=1$, the $n^{\text {th }}$ iterate of the map will have $3^{n}$ fixed points. For $n>2$, the great majority of these points will be on orbits of minimum period $n$-approximately half will be saddles, and the rest, Mobius orbits. Assuming the map is generic, all but one of these saddles will have arisen in a saddle-node bifurcation, and the snake containing the saddle will contain a cascade of attractors. Our hypotheses are not satisfied because there is a saddle at $\lambda=0$. The orbit component containing that saddle will also contain a saddle at $\lambda=1$ and, in general, will not contain a cascade. 


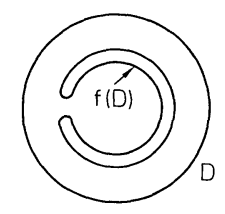

Fig. 8. A "ribbon" map $f$ of the disk into itself is shown (see Example 2, Sect. 4)

2. An example where the modified hypothesis $\left(\mathrm{H} 2^{\prime}\right)$ is useful is for area contracting maps of a disk into itself. At $\lambda=0$, there can be an attracting fixed point and no other periodic orbits. At $\lambda=1$, the map can be stretched to a ribbon and bent to fit inside the disk, as shown in Fig. 8. It is difficult to prove for a particular "ribbon" map that all orbits are unstable; but, when this is the case, the map for $0 \leqq \lambda<1$ will have at least one cascade of attractors.

Acknowledgements. We would like to thank L. Tedeschini-Lalli and S. Pelikan for their helpful suggestions.

\section{References}

[A] Alligood, K.T.: A canonical partition of the periodic orbits of chaotic maps. Trans. Am. Math. Soc. (in press)

[AMY] Alligood, K.T., Mallet-Paret, J., Yorke, J.A.: An index for the global continuation of relatively isolated sets of periodic orbits. Geometric dynamics. Lecture Notes in Mathematics, Vol. 1007, pp. 1-21. Berlin, Heidelberg, New York: Springer 1983

[AY] Alligood, K.T., Yorke, J.A.: Families of periodic orbits: virtual periods and global continuability. J. Differential Equations 55, 59-71 (1984)

[AY 2] Alligood, K.T., York, J.A.:Hopf bifurcation: the appearance of virtual periods in cases of resonance. J. Differential Equations (in press)

[B] Brunovsky, P.: (a) One parameter families of diffeomorphisms. Symposium on differential equations and dynamical systems, University of Warwick, 1969, Springer Lecture Notes 206. Berlin, Heidelberg, New York: Springer 1969; (b) On one-parameter families of diffeomorphisms. Comment. Math. Univ. Carol. 11, 559-582 (1970)

[C] Chirikov, B.V.: A universal instability of many-dimensional oscillator systems. Phys. Rep. 52, 265-379 (1979)

[CMY] Chow, S.N., Mallet-Paret, J., Yorke, J.: A bifurcation invariant: degenerate orbits treated as clusters of simple orbits. Geometric dynamic. Lecture Notes in Mathematics, Vol. 1007, pp. 109-131. Berlin, Heidelberg, New York: 1983

[DN] Devaney, R., Nitecki, Z.: Shift automorphisms in the Henon mapping. Commun. Math. Phys. 67, 137-146 (1979)

[F] Franks, J.: Period doubling and the Lefschetz formula. Trans. Am. Math. Soc. 287, 275-283 (1985)

[GH] Guckenheimer, J., Holmes, P.J.: Nonlinear oscillations, dynamical systems, and bifurcations of vector fields. Berlin, Heidelberg, New York: Springer 1983

[H] Holmes, P.J.: The dynamics of repeated impacts with a sinusoidally vibrating table. J. Sound Vib. 84 (2), 173-189 (1982)

[M] Myrberg, P.J.: Sur l'iteration des polynomes reels quadratiques. J. Math. Pures Appl. 41, 339-351 (1962)

[MY] Mallet-Paret, J., Yorke, J.: Snakes: Oriented families of periodic orbits, their sources, sinks, and continuation. J. Differential Equations 43, 419-450 (1982) 
[N] Newhouse, S.: Lectures on dynamical systems. In: Dynamical systems. Progress in Mathematics, Vol. 8. Boston: Birkhäuser 1980

[P] Peixoto, M.M.: On an approximation theorem of Kupka and Smale. J. Differential Equations 3, 214-227 (1966)

[R] Robinson, C.: Bifurcation to infinitely many sinks. Commun. Math. Phys. 90, 433-459 (1983)

[S] Smale, S.: Differentiable dynamical systems. Bull. Am. Math. Soc. 73, 747-817 (1967)

[SY] Short, T., Yorke, J.A.: Truncated development of chaotic attractors in a map when the Jacobian is not small. In: Chaos and statistical mechanics. Proc. of the 1983 Kyoto Summer Institute. Berlin, Heidelberg, New York: Springer 1983

[YA] Yorke, J.A., Alligood, K.T.: Cascades of period-doubling bifurcations: a prerequisite for horseshoes. Bull. Am. Math. Soc. 9, 319-322 (November, 1983)

[Z] Zaslovsky, G.M.: The simplest case of a strange attractor. Phys. Lett. 69A (3), 145-147 (1978)

Communicated by O. E. Lanford

Received February 21, 1984; in revised form April 16, 1985 
